\title{
Early intervention in psychosis: health economic evaluation using the net benefit approach in a real-world setting
}

Caragh Behan, Brendan Kennelly, Eric Roche, Laoise Renwick, Sarah Masterson, John Lyne, Brian O'Donoghue, John Waddington, Catherine McDonough, Paul McCrone and Mary Clarke

\section{Background}

Early intervention in psychosis is a complex intervention, usually delivered in a specialist stand-alone setting, which aims to improve outcomes for people with psychosis. Previous studies have been criticised because the control used did not accurately reflect actual practice.

\section{Aims}

To evaluate the cost-effectiveness of early intervention by estimating the incremental net benefit (INB) of an early-intervention programme, delivered in a real-world setting. INB measures the difference in monetary terms between alternative interventions.

\section{Method}

Two contemporaneous incidence-based cohorts presenting with first-episode psychosis, aged 18-65 years, were compared. costs and outcomes were measured over 1 year. The main outcome was avoidance of a relapse that required admission to hospital or home-based treatment.

\section{Results}

From the health sector perspective, the probability that early intervention was cost-effective was 0.77 . The INB was $€ 2465$ per person ( $95 \% \mathrm{Cl}-€ 4418$ to €9347) when society placed a value of $€ 6000$, the cost of an in-patient relapse, on preventing a relapse requiring admission or home care. Following adjustment, the probability that early intervention was cost-effective was 1, and the INB to the health sector was $€ 3105$ per person $(95 \% \mathrm{Cl}$ $€ 8453$ to $€ 14663)$. From a societal perspective, the adjusted probability that early intervention was cost-effective was 1 , and the INB was €19 928 per person ( $95 \% \mathrm{Cl}-€ 2075$ to €41 931).

\section{Conclusions}

Early intervention has a modest INB from the health sector perspective and a large INB from the societal perspective. The perspective chosen is critical when presenting results of an economic evaluation of a complex intervention.

\section{Keywords}

First-episode psychosis; cost-effectiveness; psychotic disorders; community mental health teams; economics.

\section{Copyright and usage}

(c) The Royal College of Psychiatrists 2019.
There has been considerable debate in both academic and health policy circles about the merits or otherwise of specialist early intervention services for people suffering from psychosis. A number of studies have found that early intervention achieves savings over time through reductions in in-patient admissions. ${ }^{1-4}$ A variety of methodological approaches have been used in previous studies. The majority used a historical treatment-as-usual (TAU) control, ${ }^{1,2,5-9}$ three used decision analytic modelling, ${ }^{10-12}$ one used data linkage ${ }^{13}$ and two used a randomised controlled trial (RCT) format. ${ }^{3,4}$ Critics have questioned whether the TAU services in some of these studies are a reasonable reflection of current practice, arguing that community mental health teams (CMHTs) are able to deliver sophisticated treatments such as home-based treatment and assertive outreach treatment. ${ }^{14,15}$ Another issue with previous studies is that those that used patientlevel data focused on younger people, whereas recent policy changes have recommended extending early intervention beyond the youth population. ${ }^{16}$ There is an urgent need, therefore, to compare the costs and outcomes of a specialist early intervention service with a TAU that routinely offers home-based and assertive outreach treatments, and to include older individuals in the analysis.

\section{Economic evaluation}

Economic evaluation is a useful method for examining whether an intervention translates into the real world, thereby generating information relevant for service planning and policy makers. Cost-effectiveness evaluations typically report incremental cost-effectiveness ratios (ICERs). However, there are difficulties in interpreting the value of the ICER and in generating a measure of uncertainty, and they are not amenable to regression analysis. ${ }^{17,18}$ Reformulating the cost-effectiveness question to generate net benefit estimates facilitates the interpretation of the results of an evaluation, and allows us to use regression techniques to adjust for sociodemographic and clinical differences between the TAU and intervention groups. Incremental net benefit (INB) measures the difference in monetary benefit between alternative interventions. A positive INB indicates that an intervention is cost-effective at a given willingness-to-pay threshold. To the best of our knowledge, only one other study has examined the cost-effectiveness of early intervention using the INB approach. However, that study compared a specialist early intervention service with a traditional TAU community model that did not deliver home-based and assertive outreach treatments. ${ }^{4}$

\section{Aim}

The aim of this study was to conduct a cost-effectiveness evaluation of early intervention in a real-world setting compared with best-practice TAU, using the INB framework. This study is presented according to the Consolidated Health Economic Evaluation Reporting Standards (CHEERS) guidelines for economic evaluations. ${ }^{19}$

\section{Method}

The study sample consisted of two incidence-based cohorts presenting to five catchment area services in the Republic of Ireland between 2010 and 2012. Data were collected at first presentation and at 1 year of follow-up. Individuals presenting with firstepisode psychosis (FEP) aged between 18 and 65 years were included. Exclusion criteria were intellectual disability, being on 
antipsychotic medication for $>30$ days before presentation and having a diagnosis of psychosis secondary to a general medical condition. The authors assert that all procedures contributing to this work comply with the ethical standards of the relevant national and institutional committees on human experimentation and with the Helsinki Declaration of 1975, as revised in 2008. Ethical approval for the study was obtained from the local ethics committees of the relevant services (identification 406 St John of God Provincial Ethics Committee). All participants gave written informed consent.

The early intervention cohort consisted of people from two urban and one predominantly rural catchment areas who were referred by their general practitioner or the local CMHT to an early intervention service, which operated as a specialist hub to three CMHT catchment areas. The early intervention service offered early detection and a phase-specific intervention strategy. ${ }^{20,21}$ Referral to the early intervention service was via primary care or the CMHT. Everybody referred was registered with a CMHT and were offered a rapid assessment within $72 \mathrm{~h}$ to establish the presence of psychosis. Where possible, the assessor also interviewed a family member. Interpreters were used where required. The CMHT was responsible for care during any in-patient admission and subsequent medication management. Each participant was offered one or all of three phase-specific interventions. Cognitive-behavioural therapy for psychosis was delivered in a group format, over 12 sessions. Family education and intervention was delivered in a group format, over six sessions. A psychosocial intervention focusing on vocational or educational needs was delivered in individual sessions for as many as were required. A followup assessment was conducted at 1 year.

The TAU cohort consisted of people from two predominantly rural catchment areas who presented to their CMHT services, which offered home-based treatment teams (HBTs) and assertive outreach teams. Patients presenting to the TAU service were assessed by a research registrar following presentation (on average within 41 days), but otherwise received standard care. ${ }^{22}$

In both cohorts, participants were assessed using the Structured Clinical Interview for DSM-5 to establish a diagnosis of psychosis. ${ }^{23}$ Information on health service and resource use was collected using the Client Socio-Demographic Service and Receipt Inventory (CSSRI) for a 1-year period. ${ }^{24,25}$ This information was supplemented by medical records and contact with the primary care service. Unit costs were derived from previously published studies and personal contact with the individual services or the finance department of the Health Service Executive. Where Irish cost data were not obtainable, we used data from the Personal Social Services Research Unit (PSSRU) and converted the costs to Euros using purchasing power parity (PPP) and the Consumer Health Index. ${ }^{26}$ Lost productivity was valued at the average industrial wage. ${ }^{27}$ Informal care was valued by the average wage of carers and child care providers based on the proxy good approach. Costs were reported in Euros for the year 2012.

The reference case takes the health sector perspective. Costs accruing to the health sector during the year following presentation with FEP were collected. These included mental health in-patient costs, general medical costs related to the FEP, home-based treatment costs, CMHT service costs, costs of primary care, and counselling, medication and investigation costs. Secondary analysis was from the societal perspective. The 1-year follow-up period allowed sufficient time for the outcome to occur. Therefore a discount rate was not applied to the costs or benefits.

The primary outcome was a relapse of psychosis sufficiently severe to require admission to hospital or the HBT. Relapse limits recovery and is distressing for the person and their family and carers. Between 30 and $70 \%$ of people with FEP will relapse. ${ }^{28}$
Information on relapse was collected from hospital electronic records and CMHT records. The outcome was recorded as a dummy variable indicating relapse or no relapse. Information on lost productivity and employment status was collected from clinical interviews and/or medical records review.

Data were analysed in Microsoft Excel 2010 and Stata version 13.0 (Windows 7). Univariate analysis of outcome data were carried out using $\chi^{2}$-tests, parametric data were analysed using Student $t$-tests, and non-parametric data were analysed using Mann-Whitney $U$ tests. Multivariate analyses of outcome data were carried out using logistic regression. As the cost data were highly skewed, cost data were analysed using a generalised linear model with a gamma family and a log link. Cost and outcome data were adjusted for sociodemographic and baseline clinical characteristics in the multivariate analyses. The net benefit statistic was generated using the equation $N B=\lambda . E-C$, where $N B$ is the net benefit, $E$ is the effectiveness (i.e. avoidance of a relapse requiring admission or HBT) and $C$ are the service costs. $\lambda$ is a theoretical unknown value placed on the outcome by society. Cost and effectiveness data were bootstrapped to 1000 replications using sampling with replacement to generate $95 \%$ confidence estimates. The proportion of replications greater than zero indicated the probability that early intervention was more cost-effective than TAU. The probabilities were used to generate cost-effectiveness acceptability curves. Sensitivity analyses of the costs and outcomes were conducted.

\section{Results}

Of 307 people presenting to services who fulfilled the inclusion criteria, 270 were eligible for follow-up at 1 year, 212 were assessed at 1 year and 201 people were included in the cost-effectiveness analysis. The reasons for non-inclusion in the cost-effectiveness analysis were incomplete Client Socio-Demographic Service and Receipt Inventory data $(n=12)$ or attendance at private in-patient services only $(n=2)$. Older people were more likely to be followed up $(P=0.01)$. Table 1 shows the sociodemographic and clinical characteristics of the sample. There were no statistically significant differences in baseline characteristics as regards gender, marital status, living independently at presentation, the proportion possessing a medical card, the proportion born in Ireland or the proportion with English as their first language. The TAU cohort were younger at presentation (28 years $v .33$ years, $z=-2.646$, $P=0.008)$, and had a higher proportion in employment at baseline ( $47 \%$ v. $27 \%, \chi^{2}=7.823$, d.f. $\left.1, P=0.005\right)$. More of the early intervention cohort lived in urban areas $\left(98 \% v .39 \%, \chi^{2}=87.34\right.$, d.f. 1 , $P<0.001)$. The TAU cohort were more likely to live in areas with higher levels of deprivation (decile $9 v$. decile $4, z=5.554, P<0.001$ ).

\section{Cost-effectiveness results}

From the health sector perspective, the intervention dominated (Table 2) since it cost $€ 1681$ (s.e. €3247) less and more relapses were avoided (0.10; s.e. 0.06). The unadjusted ICER was $€ 17078$ saved per relapse avoided. The bootstrapped estimates of the ICER show that the intervention dominated in $63 \%$ of replications. Following adjustment for sociodemographic and clinical characteristics, early intervention dominated in 95\% of replications (Fig. 1). From a societal perspective, the intervention dominated in $74 \%$ of replications. Following adjustment for sociodemographic and clinical characteristics, the intervention dominated in $95 \%$ of replications. The unadjusted ICER was $€ 25543$ saved per relapse avoided. The confidence intervals were wide and not significant. From both perspectives, there were a small number of replications 


\begin{tabular}{|c|c|c|c|c|c|}
\hline $\begin{array}{l}\text { Categorical variables, } \\
n(\%)\end{array}$ & Total $(n=201)$ & $\mathrm{TAU}(n=77)$ & Early intervention $(n=124)$ & Statistic & $P$ value \\
\hline Male & $113(56)$ & $48(62)$ & $65(52)$ & 1.898 & 0.168 \\
\hline Never married & $123(61)$ & $50(65)$ & $73(59)$ & 0.736 & 0.391 \\
\hline Living independently & $135(67)$ & $82(68)$ & $53(67)$ & 0.008 & 0.930 \\
\hline Finished high school equivalent & $136(68)$ & $48(64)$ & $88(71)$ & 1.049 & 0.306 \\
\hline Employed & $70(35)$ & $36(47)$ & $34(27)$ & 7.823 & 0.005 \\
\hline Urban & $151(75)$ & 30 (39) & $121(98)$ & 87.347 & $<0.001$ \\
\hline Medical card ${ }^{a}$ & $119(59)$ & $51(66)$ & $68(55)$ & 2.554 & 0.110 \\
\hline ROI born & $153(76)$ & $59(77)$ & $94(76)$ & 0.017 & 0.895 \\
\hline SSD & $112(56)$ & $41(53)$ & $71(57)$ & 0.309 & 0.578 \\
\hline Under 35 years at presentation & $127(63)$ & $56(73)$ & $71(57)$ & 4.887 & 0.027 \\
\hline \multicolumn{6}{|l|}{$\begin{array}{l}\text { Continuous variables } \\
\text { Median (IQR) }\end{array}$} \\
\hline Age at presentation, yr & $32(24-42)$ & $28(23-38)$ & $33(27-43)$ & -2.646 & 0.008 \\
\hline Deprivation Index & $7(2-9)$ & $9(6-10)$ & $4(1-9)$ & 5.454 & $<0.001$ \\
\hline SFI & $9(7-10)$ & $8(4-9)$ & $9(8-10)$ & -4.351 & $<0.001$ \\
\hline GAF at baseline & $30(25-35)$ & 27 (23-32) & $30(25-38)$ & -2.781 & 0.005 \\
\hline
\end{tabular}

in the south-west quadrant, indicating that the intervention was both less effective and less costly than TAU. As the number of such replications is so small (3\%), we do not consider this outcome as a realistic possibility.

The intervention yielded an INB to the health sector of $€ 1796$ (s.e. €3376). This fell to $€ 1200$ (s.e. $€ 5410$ ) following adjustment for sociodemographic and clinical characteristics, even when society placed no value on avoiding a relapse requiring admission or HBT. When a value of $€ 6000$, the approximate cost of an inpatient relapse, was placed on avoiding such a relapse, the intervention resulted in an INB of approximately $€ 2465$ (s.e. €3389) to the health sector, which rose to $€ 3105$ (s.e. $€ 5890$ ) following adjustment for sociodemographic and clinical characteristics. The standard errors of the mean were large and the $95 \%$ confidence intervals were wide and not significant, reflecting a high degree of uncertainty around the cost data. When the value of $\lambda$ was $€ 0$, the probability that the intervention was cost-effective was 0.71 in the unadjusted model. Following adjustment for sociodemographic and clinical characteristics, the probability that early intervention was costeffective fell to 0.59 .

From a societal perspective, the intervention resulted in an INB of $€ 34694$ (s.e. €8994). This fell to $€ 17604$ (s.e. $€ 10$ 933) following adjustment for sociodemographic and clinical characteristics, even when society placed no value on avoiding a relapse requiring admission or HBT. When $\lambda$ was valued at $€ 6000$, implementing the intervention resulted in an INB to society of $€ 35363$ (s.e. $€ 8081$ ), or $€ 19928$ (s.e. $€ 11212$ ) following adjustment for sociodemographic and clinical characteristics. The probability that the intervention was cost-effective was 1, both before and after adjustment for sociodemographic and clinical characteristics. Supplementary Fig. 1, available at https://doi.org/doi:10.1192/bjp.2019.126, shows the probabilities that the intervention was cost-effective for a range of values of willingness to pay $(\lambda)$.

A number of sensitivity analyses were carried out. Varying the proportion of relapses to $25 \%$ (the minimum relapse rate aimed for in the Initiative to Reduce the Impact of Schizophrenia (IRIS) guidelines ${ }^{29}$ ) and adjusting for baseline costs had no effect on the results. Neither did restricting the analysis to those seen by clinical interview compared with those whose data was extracted from medical records. Including medical card status as one of the control variables had no effect on the results.

We identified a number of subgroups a priori for further analysis because of their relevance to policy makers and service managers. The results are presented in Table 3. Many early intervention services restrict by age (usually $15-35$ years) and diagnosis (usually functional psychosis). We found that delivering early intervention to the functional psychosis (schizophrenia spectrum disorder) subgroup was highly cost-effective from both a health sector and a societal perspective. This was because the proportion of relapses was higher in this subgroup in the TAU cohort $(0.18$ $v$. 0.10). As the net benefit is affected more by costs when the effect size approaches zero, a bigger effect size in this subgroup demonstrated a different INB profile.

By contrast, restricting the analysis to the age 18-35 years subgroup revealed that early intervention was less likely to be costeffective from the health sector perspective. The INB favoured traditional CMHT until the willingness to pay to avoid a relapse was over $€ 15000$. However, the INB favoured the early intervention service from the societal perspective even when the willingness to pay to avoid a relapse was $€ 0$.

\begin{tabular}{|c|c|c|c|c|}
\hline Mean (s.e.) & $\operatorname{TAU}(n=77)$ & Early intervention $(n=124)$ & Difference $^{a}$ & $95 \% \mathrm{Cl}(\mathrm{N})^{\mathrm{a}}$ \\
\hline \multicolumn{5}{|l|}{ Health sector perspective } \\
\hline Cost, $€$ & 23862 (2835) & 22181 (1857) & $-1681(3247)$ & -4721 to 8083 \\
\hline Effect (relapse avoided) & $0.74(0.50)$ & $0.84(0.03)$ & $0.10(0.06)$ & -0.21 to 0.02 \\
\hline ICER health sector & & & -17078 & \\
\hline \multicolumn{5}{|l|}{ Societal perspective } \\
\hline Cost, $€$ & 25554 (2823) & 22707 (1863) & $-2846(3246)$ & -3768 to 9018 \\
\hline Effect (relapse avoided) & $0.74(0.50)$ & $0.84(0.03)$ & $0.10(0.06)$ & -0.21 to 0.02 \\
\hline ICER societal & & & -25543 & \\
\hline
\end{tabular}



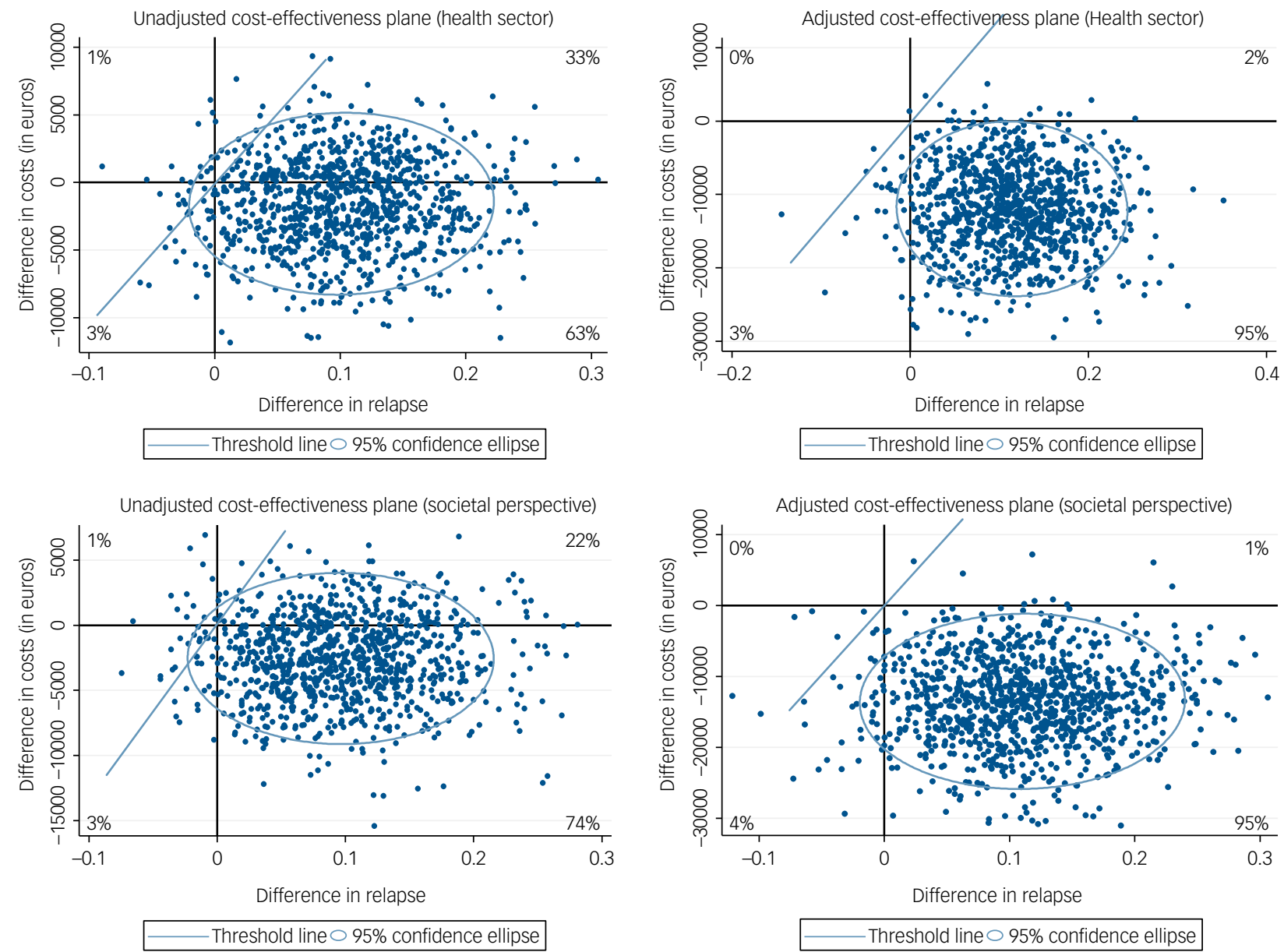

Fig. 1 Cost-effectiveness plane.

The final subgroup analysis tested the effect of including people with organic psychosis, or psychosis secondary to a general medical condition who have typically not been included in research on early intervention, despite the fact that people with psychosis secondary to a general medical condition will often present to early intervention services. Including the costs of treating the physical health cause of psychosis is reasonable as in a clinical setting it is not always immediately apparent that the cause of psychosis is medical rather than functional. These cases can generate high costs and the effect of early intervention is uncertain, as the psychosis is often resolved by treating the underlying medical condition. In our sample, although there were only four patients who presented

\begin{tabular}{|c|c|c|c|c|}
\hline When $\lambda=€ 6000$ & Reference case $(n=201)$ & Under 35 years $(n=128)$ & $\operatorname{SSD}(n=120)$ & Including GMC $(n=205)$ \\
\hline \multicolumn{5}{|l|}{$\begin{array}{l}\text { Health sector perspective } \\
\text { Unadjusted }\end{array}$} \\
\hline$\Delta \mathrm{INB}, €$, all mean (s.e.) ${ }^{\mathrm{a}}$ & 2465 (3389) & $-864(3889)$ & $7642(5433)$ & $970(3618)$ \\
\hline $95 \% \mathrm{Cl}^{\mathrm{a}}$ & -4418 to 9347 & -8497 to 6768 & -3017 to 18302 & -6130 to 8071 \\
\hline $\begin{array}{l}\text { Probability cost-effective health sector } \\
\text { Adjusted }^{\text {b }}\end{array}$ & 0.77 & 0.64 & 0.93 & 0.60 \\
\hline$\Delta \mathrm{INB}, €$, all mean (s.e.) ${ }^{\mathrm{a}}$ & $3105(5890)$ & $231(7754)$ & $6899(8712)$ & $721(5966)$ \\
\hline $95 \% \mathrm{Cl}^{\mathrm{a}}$ & -8453 to 14663 & -14985 to 15447 & -10197 to 23995 & -10985 to 13125 \\
\hline $\begin{array}{l}\text { Probability cost-effective health sector } \\
\text { Societal perspective }\end{array}$ & 1 & 0.987 & 0.998 & 0.99 \\
\hline \multicolumn{5}{|l|}{$\begin{array}{l}\text { Societal perspective } \\
\text { Unadjusted }\end{array}$} \\
\hline$\Delta \mathrm{INB}, €$, all mean (s.e.) ${ }^{a}$ & $35363(8081)$ & 34882 (10 962) & 49611 (11459) & 33582 (8028)- \\
\hline $95 \% \mathrm{Cl}^{\mathrm{a}}$ & $17582-53144$ & $12683-57134$ & $22444-76777$ & $15897-51267$ \\
\hline $\begin{array}{l}\text { Probability cost-effective societal } \\
\text { Adjusted }^{\text {b }}\end{array}$ & 1 & 1 & 1 & 1 \\
\hline$\Delta \mathrm{INB}, €$, all mean (s.e.) ${ }^{\mathrm{a}}$ & 19928 (11212) & 22509 (15 603) & 21977 (17 188) & 16779 (10977) \\
\hline $95 \% \mathrm{Cl}^{\mathrm{a}}$ & -2075 to 41931 & -8109 to 53127 & -11752 to 55705 & -4762 to 38319 \\
\hline Probability cost-effective societal & 1 & 1 & 0.996 & 1 \\
\hline
\end{tabular}


with an organic psychosis (two from each cohort), the probability that early intervention was effective from a healthcare perspective fell substantially when they were included. See Table 3 for details of the subgroup analysis.

\section{Discussion}

Our analysis of early intervention in a real-world setting found that, compared with TAU, early intervention contributed a modest benefit to the health sector and a large benefit to society. The probability that early intervention was cost-effective from a societal perspective was 1 in the reference case and in most of the subgroup analysis. Our results highlight the need to look beyond the health sector when evaluating interventions such as early intervention. Our measure of lost productivity may well be an underestimate because it did not include days in education, or measure productivity losses by carers.

Although early intervention is part of the core mental health service in Canada, England and Australia, implementation is far from uniform across regions. Other countries trying to implement early intervention face significant challenges, not least how to finance and sustain services. Another challenge is the often strict delineation between child and adolescent mental health services and general adult mental health services, with no models of care for early intervention programmes that cross this divide. This study examined whether early intervention was cost-effective in a setting where there is no transitional youth model, and early intervention is delivered using a specialist hub within a community mental health setting to an adult population aged over 18 years. Early intervention services have traditionally been restricted to younger people, yet there are a substantial proportion of people who present for care for the first time over the age of 45 years. Our study included older people (up to 65 years) in the analysis. The oldest age at onset was 63 years in both cohorts. This is a particularly relevant question from a policy perspective, as the 'Early Intervention Access and Waiting Time Standard' published in 2016 is being widely implemented in England, ${ }^{16}$ and the National Clinical Programme in Ireland Model of Care is also delivered up to 65 years of age.

The importance of restricting our analysis by age was evident in our subgroup findings, where early intervention was much less likely to be cost-effective when we excluded people aged over 35 years. Like many other countries, there is a strict boundary in the Republic of Ireland between child and adolescent mental health services and adult mental health services, a boundary that straddles the age range in which many mental health disorders manifest themselves for the first time. Our finding that early intervention services are less likely to be cost effective in the under 35 years age group should be interpreted with this caveat in mind.

The study has a number of strengths. Previous studies of early intervention have compared early intervention with older models of TAU. By contrast, the TAU cohort in this study received bestpractice community mental healthcare, including HBT and assertive community outreach, which are both designed to deliver acute care in the community. We also used a robust methodology for the case finding and evaluation of people presenting with FEP. Apart from the possibility that some patients in the areas covered by our analysis might have attended a private mental hospital for which we did not have data, both samples are epidemiological samples of FEP presenting to community mental health services in the five catchment areas included in the study. Each person attending the early intervention service received a comprehensive diagnostic interview and assessment by trained assessors with good interrater reliability. Of those presenting to the TAU catchment area, $80 \%$ were assessed at baseline with a comprehensive diagnostic interview and assessment by trained assessors, also with good interrater reliability.

The patient-level costs included in this study were collected in a structured, standardised manner. As a year is a long time for patient recall, the information provided by patients was supplemented with information from medical records and by contacting primary care practices. Evidence shows that using patient recall alone underestimates resource use and therefore costs. ${ }^{30}$

As regards limitations, the study design is not as robust as that of an RCT and there are potential sources of observed and unobserved differences between the cohorts, which might bias the results. The TAU cohort was younger and from a predominantly rural and more deprived setting with higher levels of unemployment. The early intervention cohort was a mixture of individuals from a predominantly affluent area with a relatively older population and a smaller number from deprived or rural areas. Ethnicity was not examined in this study. Although the overall trend nationally has been an inward migration of other nationalities to the Republic of Ireland over the time period between the two studies, the population has remained relatively homogenous, particularly in the catchment areas examined. We could not use duration of untreated psychosis as a covariate as this was measured differently in each cohort.

We considered using propensity score matching to simulate the conditions of an RCT design. ${ }^{31}$ However, the optimal conditions for propensity score matching usually require more observations in the control group than the treatment group. In this case there were more observations in the treatment group and using propensity score matching would have excluded a large number of people from the analysis. The propensity match score was used as a covariate in the initial analysis; however, as this did not yield any extra information in comparison to including the covariates themselves, the score was ultimately not included in the final model.

The healthcare service in the Republic of Ireland is a complex, hybrid system funded by taxation, private health insurance and out-of-pocket expenditure. A total of $40 \%$ of the population possess a medical card, which means they have access to free public healthcare (both primary and hospital), but there are very long waiting lists in the public system, particularly for elective services. The rest of the population are entitled to use the public hospital system, albeit with some co-payments. That segment of the population must pay for primary healthcare. Almost $50 \%$ of the population have supplementary private health insurance, which enables them to access acute hospital services in the public sector with less delay and to use privately operated hospitals. ${ }^{32}$ Most mental health services in the Republic of Ireland are delivered publicly by the Health Service Executive, although there are two private hospitals that specialise in mental disorders. We excluded any person who was admitted to either of the private mental hospitals during the course of the study. The two services that form the basis for this study, the early intervention and TAU, are both publicly funded services and free to the individual at the point of use. It is very unlikely that having private health insurance has any effect on a person's access to either of these services.

The Republic of Ireland does not yet have a reliable comprehensive national source for unit cost data. The possibility remains that some of the costs used were biased but it is difficult to say a priori the direction of any such bias. The standard outcome measure of choice in economic evaluations is the quality-adjusted life-year (QALY). One advantage of the QALY is that the cost per QALY threshold in any country provides an obvious estimate of society's willingness to pay for a QALY. ${ }^{18}$ In choosing a primary outcome for this study, we were restricted to one that could be reliably extracted from clinical records at 1 year. This is a typical limitation of doing research in a real-world setting. 
There was potential selection bias in the 1-year follow-up data. Because of differences in the ethical approval from each ethics committee, the tracing procedures in each cohort were different. Not all of the eligible early intervention sample was followed up at 1 year, whereas almost all the TAU sample had follow-up at 1 year either by clinical interview or by medical records. The effect of this potential selection bias was tested by examining the total sample in the reference case analysis, and then by reconducting the analysis only in those followed up by clinical interview in both samples. There were no statistically significant different sociodemographic or clinical characteristics between those followed up by clinical interview and those followed up by clinical record. In addition, there were no statistically significant differences in the primary outcome measure. There were some differences in the cost data, and therefore in the net benefit statistic in the repeat analysis. The probability that early intervention was cost-effective shifted down and to the right; however, the trajectory of the net benefit statistic remained the same. As previously alluded to, the literature suggests that patient reports of health service resource use are often higher than resource use taken from medical records, so this suggests that the cost data in the overall sample is an underestimate of the costs in the TAU group rather than an overestimate.

This study adds to the evidence base on the economic evaluation of early intervention in psychosis. Using patient-level data from a mix of urban and rural settings, the evaluation of an intervention taking place in a real-world setting with due consideration of the context in which the study took place provide additional insights into the effects of early intervention services. Previous research found that early intervention makes its cost savings by reducing in-patient admissions. Modelling studies have shown that the societal effect of early intervention is larger than its effect on the health sector through the effects early intervention has on employment and education. ${ }^{12}$ This study has provided evidence using patient-level data that early intervention, delivered in a real-world setting, in a mental health system that has no youth oriented specialist early intervention service, can still provide a modest INB to the health sector even when the value of preventing a relapse requiring admission is unknown. We also found that early intervention has a large INB and is extremely likely to be cost-effective when a societal perspective is taken. As mental health interventions will often affect outcomes outside the health service, such as employment, housing and education, policy makers and service planners should be aware of this, and consider alternate sources of funding mental health interventions as benefits accrue beyond the health service.

\footnotetext{
Caragh Behan (D), MRCPsych, PhD, Clinical Research Fellow, Dublin and East Treatment and Early Care Team (DETECT); and Clinical Research Fellow, School of Medicine, University College Dublin, Ireland; Brendan Kennelly, BA, MSC, Lecturer and University College Dublin, Ireland; Brendan Kennelly, BA, MSC, Lecturer and
Programme Director (Health Economics MSC), Department of Economics, National University of Ireland Galway, Ireland; Eric Roche, MRCPsych, PhD, Clinical Research
Fellow, DETECT; and Clinical Research Fellow, School of Medicine, University College Dublin, Ireland; Laoise Renwick, PhD, Clinical Nurse Specialist, HRB Nursing and Midwifery Fellow, DETECT, Ireland; Sarah Masterson, MSC, Research Assistant, DETECT, Ireland; John Lyne, MRCPsych, PhD, Consultant Psychiatrist, Beaumont Hospital, Dublin; and Honorary Senior Lecturer, Department of Psychiatry, Royal College of Surgeons in Ireland, Ireland; Brian O'Donoghue, MRCPsych, PhD, senior Research of Surgeons in Ireland, Ireland; Brian O'Donoghue, MRCPSych, PhD, Senior Researt
Fellow, Orygen National Centre of Excellence in Youth Mental Health; and Senior Fellow, Orygen National Centre of Excellence in Youth Mental Health; and Senior
Research Fellow, University of Melbourne, Australia; John Waddington, PhD, DSc, MRIA, Professor of Neuroscience, Faculty of Medicine and Health Sciences, Molecular and Cellular Therapeutics, Royal College of Surgeons in Ireland, Ireland; Catherine McDonough, MD, FRCPsych, Consultant Psychiatrist and Clinical Lead, COPE Early Intervention Service, Cavan and Monaghan Mental Health Services, Ireland; Paul Mccrone, PhD, Professor of Health Economics, King's Health Economics, Institute of Psychiatry, Psychology \& Neuroscience, King's College London, UK; Mary Clarke, MD, FRCPI, FRCPsych, Clinical Lead, DETECT; and Associate Clinical Professor of Psychiatry, Department of Psychiatry, School of Medicine, University College Dublin, Ireland
}

Correspondence: Caragh Behan, DETECT Early Intervention Service, Avila House, Block 5, Blackrock Business Park, Blackrock, County Dublin A94 P6H3, Ireland.

Email: clbehan@gmail.com

First received 23 Nov 2018, accepted 30 Apr 2019

\section{Supplementary material}

Supplementary material is available online at https://doi.org/10.1192/bjp.2019.126.

\section{Funding}

This study was funded by Health Research Board (HRB) grant HPF-2011-042. Funding for dissemination was received from the Discipline of Economics, NUIG, the School of Economics, NUIG, Brendan Kennelly, and the St John of God Research Foundation. There was no conflict of interests to report. The Principal Investigator of the HRB grant also worked in the early intervention service.

\section{References}

1 Mihalopoulos C, McGorry PD, Carter RC. Is phase-specific, communityoriented treatment of early psychosis an economically viable method of improving outcome? Acta Psychiatr Scand 1999; 100(1): 47-55.

2 Mihalopoulos C, Harris M, Henry L, Harrigan S, McGorry P. Is early intervention in psychosis cost-effective over the long term? Schizophr Bull 2009; 35(5): 909-18.

3 Hastrup LH, Kronborg C, Bertelsen M, Jeppesen P, Jorgensen P, Petersen L, et al. Cost-effectiveness of early intervention in first-episode psychosis: economic evaluation of a randomised controlled trial (the OPUS study). $\mathrm{Br} J$ Psychiatry 2013; 202(1): 35-41.

4 Mccrone P, Craig TK, Power P, Garety PA. Cost-effectiveness of an early intervention service for people with psychosis. Br J Psychiatry 2010; 196(5): 377-82.

5 Cullberg J, Mattsson M, Levander S, Holmqvist R, Tomsmark L, Elingfors C, et al. Treatment costs and clinical outcome for first episode schizophrenia patients: a 3-year follow-up of the Swedish 'Parachute Project' and two comparison groups. Acta Psychiatr Scand 2006; 114(4): 274-81.

6 Goldberg K, Norman R, Hoch JS, Hoch J, Schmitz N, Windell D, et al. Impact of a specialized early intervention service for psychotic disorders on patient characteristics, service use, and hospital costs in a defined catchment area. Can J Psychiatry 2006; 51(14): 895-903.

7 Wong KK, Chan SKW, Lam MML, Hui CLM, Hung SF, Tay M, et al. Costeffectiveness of an early assessment service for young people with early psychosis in Hong Kong. Aust N Z J Psychiatry 2011; 45(8): 673-80.

8 Behan C, Cullinan J, Kennelly B, Turner N, Owens E, Lau A, et al. Estimating the cost and effect of early intervention on in-patient admission in first episode psychosis. J Ment Health Policy Econ 2015; 18(2): 57-62.

9 Cocchi A, Mapelli V, Meneghelli A, Preti A. Cost-effectiveness of treating firstepisode psychosis: five-year follow-up results from an Italian early intervention programme. Early Interv Psychiatry 2011; 5(3): 203-11.

10 Serretti A, Mandelli L, Bajo E, Cevenini N, Papili P, Mori E, et al. The socioeconomical burden of schizophrenia: a simulation of cost-offset of early intervention program in Italy. Eur Psychiatry 2009; 24(1): 11-6.

11 McCrone $P$, Knapp M, Dhanasiri S. Economic impact of services for firstepisode psychosis: a decision model approach. Early Interv Psychiatry 2009; 3(4): 266-73.

12 Park AL, McCrone P, Knapp M. Early intervention for first-episode psychosis: broadening the scope of economic estimates. Early Interv Psychiatry 2014; 2: 144-51

13 Tsiachristas A, Thomas T, Leal J, Lennox BR. Economic impact of early intervention in psychosis services: results from a longitudinal retrospective controlled study in England. BMJ Open 2016; 6(10): e012611.

14 Castle DJ. The truth, and nothing but the truth, about early intervention in psychosis. Aust N Z J Psychiatry 2012; 46(1): 10-3.

15 Bosanac $P$, Patton GC, Castle DJ. Early intervention in psychotic disorders: faith before facts? Psychol Med 2010; 40(3): 353-8.

16 National Collaborating Centre for Mental Health; National Institute for Health and Care Excellence. Implementing the Early Intervention in Psychosis Access and Waiting Time Standard: Guidance. NHS England, 2016 (https://www. england.nhs.uk/mentalhealth/wp-content/uploads/sites/29/2016/04/eipguidance.pdf).

17 Hoch JS, Briggs AH, Willan AR. Something old, something new, something borrowed, something blue: a framework for the marriage of health econometrics and cost-effectiveness analysis. Health Econ 2002; 11(5): 415-30.

18 Drummond MF, Sculpher MJ, Claxton K, Stoddart GL, Torrance GW. Methods for the Economic Evaluation of Health Care Programmes (4th edn). Oxford University Press, 2015.

19 Husereau D, Drummond M, Petrou S, Carswell C, Moher D, Greenberg D, et al. Consolidated Health Economic Evaluation Reporting Standards (CHEERS) statement. BMJ 2013; 346: f1049. 
20 Lyne JP, O'Donoghue B, Roche E, Behan C, Jordan I, Renwick L, et al. Illness characteristics and symptoms in an Irish early intervention for psychosis service. Ir J Psychol Med 2015; 32(1): 147-54.

21 O'Donoghue B, Lyne J, Kinsella A, Turner N, O'Callaghan E, Clarke M. Detection and characteristics of individuals with a very long duration of untreated psychosis in an early intervention for psychosis service. Early Interv Psychiatry 2014; 8(4): 332-9.

22 Baldwin P, Browne D, Scully PJ, Quinn JF, Morgan MG, Kinsella A, et al. Epidemiology of first-episode psychosis: illustrating the challenges across diagnostic boundaries through the Cavan-Monaghan study at 8 years. Schizophr Bull 2005; 31(3): 624-38.

23 First MB, Spitzer RL, Gibbon M, Williams JB. Structured Clinical Interview for DSM-IV-TR Axis I Disorders, Research Version, Non-Patient Edition (SCID-I/NP). New York State Psychiatric Institute, 2002 (https://doi.org/10.1007/978-0-38779948-3_2011).

24 Knapp M, Beecham J. Costing mental health services. Psychol Med 1990; 20(4): 893-908

25 Chisholm D, Knapp MR, Knudsen $\mathrm{HC}$, Amaddeo F, Gaite L, van Wijngaarden $\mathrm{B}$. Client Socio-Demographic and Service Receipt Inventory-European Version: development of an instrument for international research. EPSILON Study

5. European psychiatric services: inputs linked to outcome domains and needs. Br J Psychiatry Suppl. 2000; (39): s28-33.

26 Curtis L (Editor). Unit Costs of Health and Social Care 2012. Personal Social Services Research Unit, 2012 (https://www.pssru.ac.uk/project-pages/unitcosts/unit-costs-2012/)

27 Central Statistics Office. Statistical Yearbook of Ireland 2012 Edition. Central Statistics Office, 2012 (http://www.cso.ie/en/statistics/statisticalyearbookofireland/statisticalyearbookofireland2012edition/).

28 Addington D, Addington MDJ, Patten S. Relapse rates in an early psychosis treatment service. Acta Psychiatr Scand 2007; 115(2): 126-31.

29 IRIS. IRIS Guidelines Update. IRIS Initiative Ltd, 2012 (http://www.iris-initiative. org.uk/silo/files/iris-guidelines-update-september-2012.pdf)

30 Gillespie P, O'Shea E, Smith SM, Cupples ME, Murphy AW. A comparison of medical records and patient questionnaires as sources for the estimation of costs within research studies and the implications for economic evaluation Fam Pract 2016; 33(6): 733-9.

31 Heckman JJ, Ichimura $\mathrm{H}$, Todd $\mathrm{P}$. Matching as an econometric evaluation estimator. Rev Econ Stud 1998; 65(2): 261-94.

32 Nolan A. Health: funding, access and efficiency. In The Economy of Ireland (eds J'Hagan, F O'Toole): 356-74. Palgrave, 2017. 